\title{
The Application of Agent Technology on the Power Flow of Iraqi Supper Grid Network
}

\author{
${ }^{1}$ Shant K. Avakian, ${ }^{2}$ Afaneen Anwar and ${ }^{3}$ Ahmed N. Abdalla \\ ${ }^{1}$ Department of Computer Science, University of Technology, Baghdad, Iraq \\ ${ }^{2}$ Department of Electric and Electronics Engineering, University Technology, Baghdad, Iraq \\ ${ }^{3}$ Department of Electric and Electronics Engineering \\ Huazhong University of Science and Technology, Wuhan, China
}

\begin{abstract}
A study was conducted to improve the Iraqi National Control center by adapting the facilities of the agent technology and to obtain an automated control system for controlling and monitoring the electrical network. This study presents simulation results of practical agent in power flow control scenario applied to the Iraqi super grid network $(400 \mathrm{kV})$.
\end{abstract}

Key words: Agent technology, power system control, load flow analysis

\section{INTRODUCTION}

Communication plays a very limited role in the traditional control systems, but a new area of decentralized control and efficiency demands has led to an environment demanding efficiency and reliability that pushes these legacy methods to their limits. The power system industry is beginning to recognize the benefits that communication could contribute towards greater system correctness. The pervasiveness of the internet has, at the same time, led to great interest in using communication networks for improved control systems. In particular, there has been an increasing interest within the power community in the use of networked agents to improve the reliability and efficiency of the electrical power grid $^{[1]}$.

This research has led to the development of mathematical and computational techniques that are applicable to the design of distributed decision support systems based on the model of a complex system composed of multiple, autonomous and adaptive intelligent agents. Many scholars have put forth a variety of architecture of MAS. The first comprehensive exposition of the automatic decisionmaking system can be found in literature ${ }^{[2]}$.

Ebrahim and Morison added the dynamic security analyses to the SCADA/EMS system and established respective system architectures ${ }^{[3-6]}$. Qing Zhao and Garng Huang established a power market simulation system based on two layer architecture $^{[7]}$ : market operation system (MOS) and power system operation system (PSOS).Wang Mingjun used CBR and multiagent technique to help dispatcher to make decision ${ }^{[8]}$.Wang Cheng shang and $\mathrm{Yu} \mathrm{Xu}$ yang put forth a emergency control system based on multiagent ${ }^{[9]}$.

Multi-agent technique has become the most promising method to build complex software system, but there are still some problems need further research: 1.domain concepts and models which is the living environment of agent; 2. realization method of individual agent; 3 . architecture of multi-agent system and cooperative strategy of agents.

In this study, three of the agent properties were used to develop a computer program that is autonomous, interactive and have the ability to communicate over a network.

Agent architecture: In general, the agent uses geographically distributed agents located in a number of Intelligent Electrical Devices (IEDS). An IED is a hardware environment that has the necessary computational, communication and other I/O capabilities needed to support a software agent. An IED can be loaded with agents that can perform control and/or protection functionality. Theses agent based IEDS work in an autonomous manner where they interact both in their environment and with each other.

The agents perceive more global scope of the system and act in response to their non-local environment by communication with other agents via one of the following communication networks:

* Local Area Network (LANS): A LAN is usually privately owned and links the devices in a single office, building, or camps including voice, sounds and video peripherals.

* Metropolitan Area Network (MAN): A MAN is designed to extend over an entire city. It may be a single network, or it may be means of connecting a number of LANS into a large network so that resources may be shared LAN-to-LAN.

* Wide Area Network (WAN): A WAN provides long distance transmission of data, voice, image and video information over large geographical 
areas that may comprise a country, a continent, or over the whole world.

On the other hand, agents might interface with legacy systems such as Supervisory Control and Data Acquisition (SCADA).

The power flow problem: Power flow studies are of greet importance in planning and designing the future expansion of power systems as well as in determining the best operation of existing systems and also it is used widely in power system control centers.

In power flow problem there are a number of nonlinear relationships between voltage and current at each bus which must be solved for all voltages and currents such that these nonlinear relationships are met. The complexity of obtaining a formal solution for power flow in power system arises because of differences in the type of data specified for the different type of buses. Although the formulation of sufficient equations to match the number of unknown state variables is not difficult, the closed form of solution is not practical $^{[10]}$.

There are many methods which provide digital solutions to power-flow equations that follow an iterative process, but the most sufficient method is the Fast-Decoupled method. This method offers a uniquely attractive combination of advantages over established methods, including Gauss Seidel and Newton Raphson, in terms of computational speed, reliability, simplicity and storage for power flow solutions.

The program which was used in this study apply the Fast Decoupled method written in MATLAP language and the flow-chart of this program is shown in Fig. $1^{[10]}$.

The proposed simulation structure of the communication network of the iraqi control centers: The Iraqi control system consists of ${ }^{[11]}$ :

* The National Control Center (NCC) in Baghdad.

* The regional control centers (RCC):

1. North region control center (NRCC) in Kerkuk which is responsible for controlling and monitoring the electrical network in the north region of Iraq (SDM4, MOS4, BAJ4, KRK4).

2. Middle region control center (MRCC) in Baghdad which is responsible for controlling and monitoring the electrical network in the middle region of Iraq (BGE4, BGW4, BGN4, BGS4, HAD4, QAM4, BQB4, KUT4, BAB4. and MSB4).

3. South region control center (SRCC) in khur Alzubair which is responsible for controlling and monitoring the electrical network in the south region of Iraq (KAD4, QRN4, NAS4, HRTP and KAZ4).

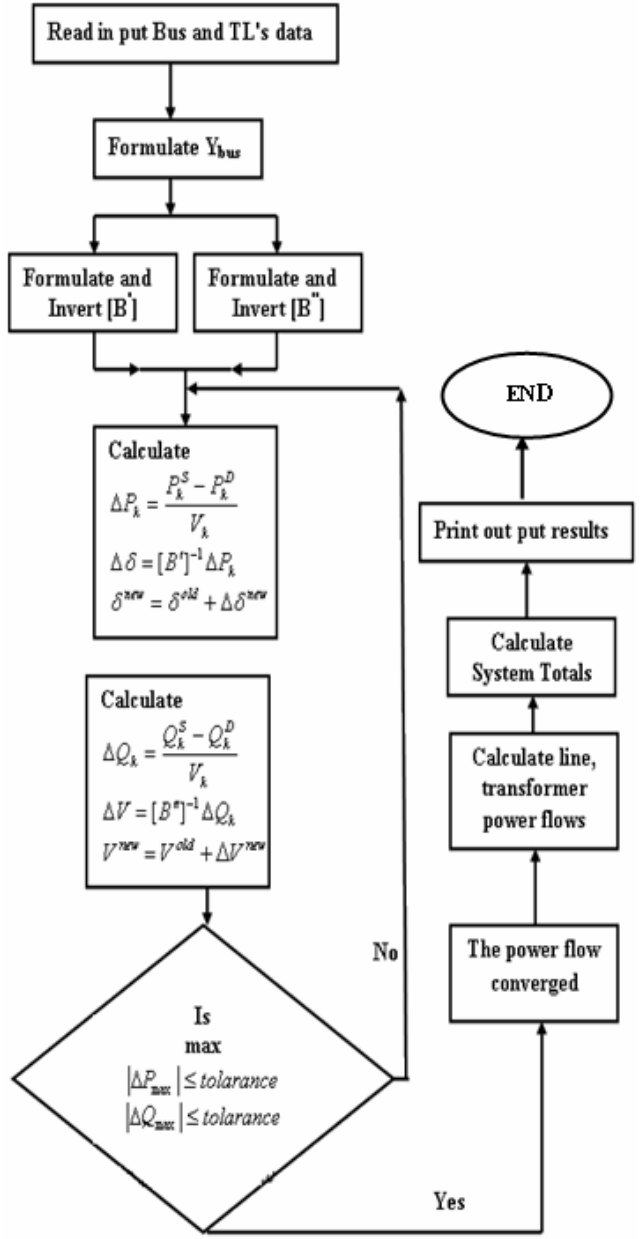

Fig. 1: Flow chart for a fast decoupled power flow $\operatorname{program}^{[10]}$

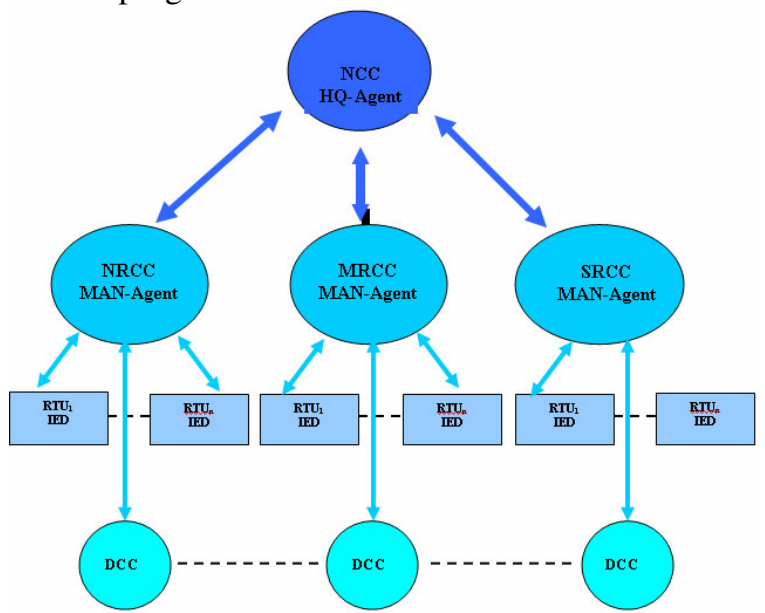

Fig. 2: The proposed agent communication network of the Iraqi control system

The distribution control centers(DCC): The traditional way of translating the information in this control system is that the information will be sent from/ to the RTU's (the remote terminal units) in each station from/ to the RCC's then from/ to the NCC where it will be analyzed off-line and the solution of any abnormal 
condition will be sent to the DCC's to take the proper action by the operators.

The new package (system) was built using Object Oriented Programming (OOP) method where the simulation of the network is made by building three classes, the first class simulates the work of the HQ agent, the second class simulates the work of the RCCs agent and the last class simulates the work of the IEDs agent. One object is created for the HQ agent, three objects are created to represent the RCCs agents and objects are created to represent the IEDs. The objects are created as threads so that they will work in parallel. Objects are able to communicate so that to simulate the information movement between The HQ, RCCs and IEDs.

The proposed structure which is shown in Fig. 2, has the ability to sense automatically any disturbance such as the separation of a transmission line, a unit generation of any generation station and the separation of a hole station by the RTUs. Since the RTUs contains the IEDs, it will translate the new data to the RCCs which contains the MAN agent, the RCC will translate information to the NCC where the HQ agent will analyze the new situation of the electrical network by running the load-flow program to obtain the new power distribution in the network.

Now the solution of the abnormal condition will be passed to the DCC to give the orders to the control and protection devices in order to overcome the problem automatically.

The application of the new program on the Iraqi super grid network: The network under consideration represents the Iraqi super grid network which consists of 19 bus-bar and 30 transmission lines, the input data to the load flow program was taken from the INDC (Iraqi National Dispatch Center) representing the load and the generation of this network on the 2nd of Jan. 2000. See the appendix.

The new program can operate within the agent environment only. Therefore, if we assume that this system is available at the Iraqi control centers, then the program can be connected directly to the electrical system and the load flow analysis can be converted from off-line application into on-line application.

At the beginning of the new program the configuration of the super grid network will appear to the user, as shown in Fig. 3 with all its bus bar and transmission lines. The bus bar will be colored according to their type (red for the generation bus bars and blue for the load bus bars and pink for the slack bus), also the value and the direction of transmission line loading will appear in green. By clicking at any bus bar a selection menu will appear to select the type of disturbance.

To exam the new program on the network under consideration, three types of disturbances were applied, those are:

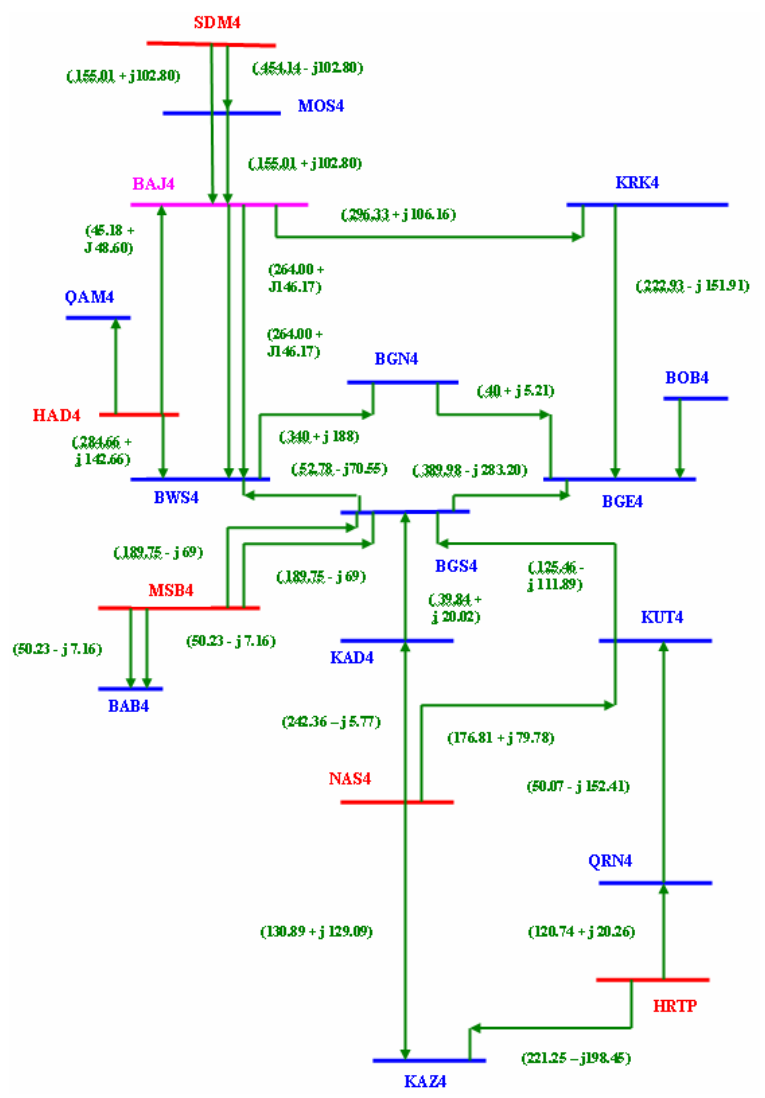

Fig. 3: The configuration of the 400- $\mathrm{kV}$ network with its loading

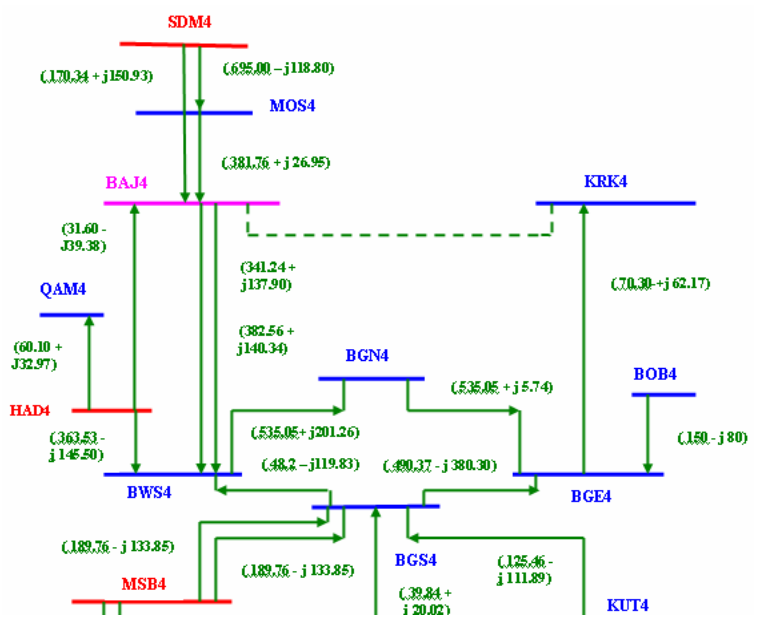

Fig. 4: The Separation of BAJ4-KRK4 transmission line

1. The separation of a transmission line: by clicking on KRK4 bus, a menu of all transmission lines connected to the selected bus appears and BAJ4-KRK4 transmission line is chosen. The agent in the NRCC assign the problem and sent the information to the HQ agent which in return run the load flow program under the new situation to calculate the new power distribution and sent it back to 


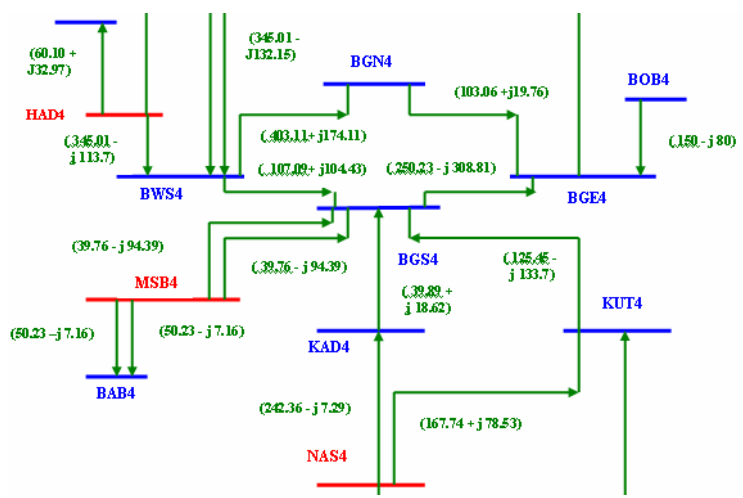

Fig. 5: The Results of the reduction MSB4 Bus's generation

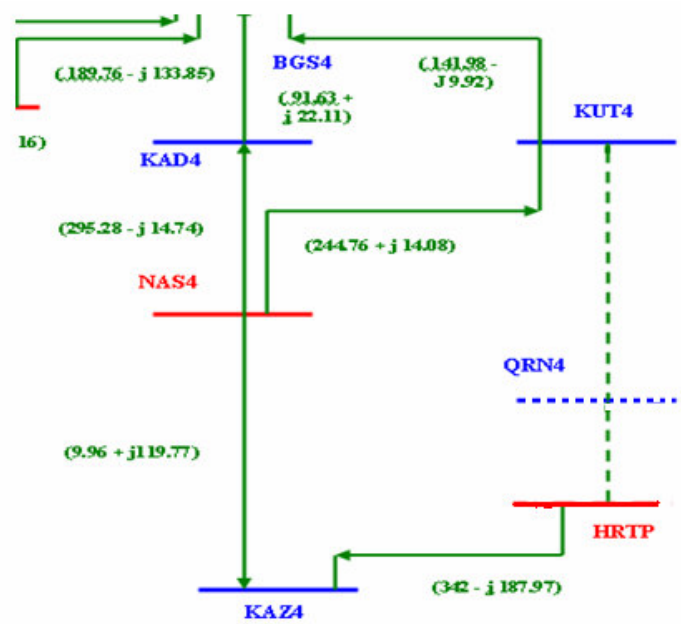

Fig. 6: The separation of QRN4 bus

the RTU's through the NRCC in order to send the protection and control orders to the stations directly. Also the new power distribution will be sent to the DCC.

2. The reduction of a generation power from a generation bus bar: the chosen bus was MUS4 bus the separated power was $200 \mathrm{MW}$. The MRCC will sense the disturbance and will send the information to the HQ agent. The load flow is run and the new power distribution is sent to the RTU's .

3. The separation of a bus bar : the chosen bus is QUR4. By choosing the fist selection from the Agent power selection window, then choose the second selection from bus bar widow, the agent in the SRCC assigns the disturbance and sent the new data to the HQ and the information is sent to the RTU's after the running of the load flow by the HQ agent.

The separation of a bus will be accompanied with the separation of all transmission lines connected to it, the type of the line and its color will be changed with the separation process. Also the type of the bus bar will be changed (from continues to a dashed line).

After each run of the load flow the results will appear on the configuration of the network with all transmission line loading.
The disturbance can be removed by clicking on the selected bus and by selecting the type of the element to be retrieved, the agent at that region will sense the changes and translate the new information to the HQ agent which in return will retrieve the element to the load flow input file, run the program and distribute the results of the power flow to the RTUs's agents through the RCCs's agents.

\section{CONCLUSION}

This study presents studies to improve the Iraqi control system by using agent technology. To form the bases of this improvement, a new control structure was proposed using agent networks to convert an off-line load flow program into on-line program. The main difference between the old control system and the new one is that the new structure has the ability not only to collect data from the electrical network as the old system do, but also to control the network automatically.

By using the proposed structure, the electrical network will be able to monitor and control more efficiently.

\section{REFERENCES}

1. James, S.T., X. Wang and K.H. Hopkinson, 2004. Agent Technology Applied to the Protection of power system. Seenering Critical Infrastructures, Grenoble.

2. Dy-Liacco, T.E, 1997. Enhancing power system security control. IEEE Trans. Computer Applications in Power, 10: 38-41.

3. Ebrahim, V., Y. Mansour and E.K. Tse, 1998. A general purpose method for on-line dynamic security assessment. IEEE Trans. Power System, 13: 243-249.

4. Ebrahim, V. and K.W. Cheung, 2000. Evolution and future of on line DSA. IEEE. Power Engineering Society Winter Meeting, 1: 63-65.

5. Jardim, J.L., 2000. Online dynamic security assessment: implementation problems and potential use of artificial intelligence. IEEE. Power Engineering Society Summer Meeting, 1: 340-345.

6. Morison, K., L. Wang and P. Kundur, 2004. Power system security assessment. IEEE. Power \& Energy Mag., 2: 30-39.

7. Zhao, Q., G. Huang, X. Wu and X. Luo, 2003. A software architecture for power market supporting system and reengineering of legacy EMS. IEEE Trans. Power Systems, 18: 191-197.

8. Mingjun, W., 2004. Application of case based reasoning and multi-agent in power system security control. Power System Technology, 28: 1-5.

9. Chengshan, W. and Y. Xuyang, 2004. Distributed coordinative emergency control based on multi-agent system. Power System Technology, 28: 1-5.

10. Anwar, A., 2005. Implementation of Geographic Information system (GIS) in Transient Stability Assessment. M.Sc. Thesis, University of Technology, Electrical and Electronic Department, Baghdad, Iraq.

11. The Iraqi National Dispatch Center (NDC), 2005. 